\title{
The Effects of Watching a Virtual Reality (VR) Forest Video on Stress Reduction in Adults
}

\author{
Sungjun Hong, Dawou Joung, Jeongdo Lee, Da-Young Kim, Soojin Kim, and Bum-Jin Park ${ }^{*}$ \\ Department of Environment \& Forest Resources, Chungnam National University, Daejeon 34134, Korea
}

\begin{abstract}
This study was conducted to investigate the effects of watching a virtual reality (VR) forest video on stress reduction in adults. Experiments were performed in an artificial climate chamber where temperature, humidity and illumination were controlled. To cause stress in subjects, the subjects were asked to multiply two digit numbers by one digit numbers for two minutes. The subjects then watched a VR forest video for five minutes. During the experiment, the heart rate variability (HRV) and heart rate (HR) of subjects were continuously measured for evaluating their physiological state. After measuring the baseline and watching a VR forest video, their psychological state was evaluated using the profile of mood state (POMS), semantic differential (SD) method, and positive affect and negative affect schedule (PANAS). The results of physiological evaluation after watching a VR forest video showed a decrease in the stress index and HR, an increase in the activity of the parasympathetic nervous system, and the standard deviation of normal to normal RR intervals (SDNN). The results of psychological assessment confirmed that watching a VR forest video induced "comfort", "natural", and "soothed" feelings in subjects. In addition, watching a VR forest video decreased "tension-anxiety (T-A)", "depression-dejection (D)", "anger-hostility (A-H)", "fatigue (F)", "total mood disturbance (TMD)", and increased "vigor (V)" compared to the base line, and decreased "negative affect" and increased "positive affect." These results indicate that watching a VR forest video decreases adults' stress index, stabilizes physiological state, and has a positive impact on psychological state. The findings of this study can serve as a basis for providing forest welfare services to people in the blind spots of forest welfare, and can lay a foundation for the adoption of VR, one of the technologies of the fourth industrial revolution in forestry.
\end{abstract}

Keywords: forest landscape, forest therapy, forest welfare, heart rate variability, profile of mood state

\section{Introduction}

As people's interest in health increases, the demand for forest therapy has been also on the rise. To meet the growing demand, the Korea Forest Service (KFS) announced the G7 Project for forest welfare by life cycle stage as its major plan from 2014 to 2017 (Korea Forest Service, 2014). Forest welfare by life cycle stage is a method to reduce discrimination based on class by providing differentiated and systematic programs for each life cycle stage to ensure everyone can enjoy the benefits of forest welfare (Yeom, 2015). Under the G7 Project for forest welfare, the Korea Forest Service

This study was supported by the forest science technology R\&D project (2018128B10-1819-AB01) of the Korea Forest Service (Korea Forestry Promotion Institute).

Received: April 3, 2019, Revised: April 19, 2019, Accepted: May 21, 2019

First author: Sungjun Hong, E-mail: hsj48781880@gmail.com, (1) https://orcid.org/0000-0002-4347-704X

*Corresponding author: Bum-Jin Park, E-mail: bjpark@cnu.ac.kr, (ㄱ) https://orcid.org/0000-0003-2888-287X 
developed forest welfare programs by life cycle stage including forest prenatal education, forest experience centers for children, forest education, forest trails and leisure sports, natural recreation forests, healing forests and natural burials, and has built forest welfare facilities to provide such programs (Korea Forest Service, 2018). In addition, expert training courses such as forest therapist, forest guide for children and forest tracking have been also operated (Korea Forest Service, 2018), which encourage more people to visit and enjoy forests in various ways.

However, since these forest welfare programs by life cycle stage are conducted all within forest welfare facilities located in forests, people have to visit forests to participate in the programs. Thus, people with walking difficulties who cannot easily get to forests have relatively few opportunities to enjoy forest welfare, and teenagers who fear forests due to fear of forests or insectophobia do not have a chance to visit forests, so there are relatively few opportunities to enjoy forest welfare benefits. Therefore, a method of providing forest welfare is needed to clear blind areas of forest welfare. The Korea Forest Service signed a partnership agreement with KIA Motors in 2018 to provide those with difficulties in walking with vehicles. It has also implemented a universal design for the disabled in forest recreation facilities, but areas accessible by people with difficulties in walking in mountainous areas with steep slopes are still limited (Korea Forest Service, 2019). and There is no policy for adolescents with fear of forests.

As people's interest in forest therapy increases, many studies have been conducted on the effects of forest therapy. Park (2010) reported that walking in forest environments for 15 minutes significantly increased the activity of the parasympathetic nervous system and significantly reduced the activity of the sympathetic nervous system and the concentration of stress hormones compared to walking in urban environments. Jung et al. (2015) also reported that viewing forest landscapes increased the In (HF) substance and reduced the mean heart rate compared to urban landscapes. Lee et al. (2018) reported that the forest therapy program of three days and two nights was effective in reducing the stress level of emotional labors, increasing positive emotions and decreasing negative emotions.

Unlike these studies conducted in outdoor environments, some studies were conducted in indoor environments. Gen (2015) reported that watching forest videos after being under visual stress increased tension or stability depending on the components of videos, and Alvarsson et al. (2010) reported that listening to natural sounds after being under stress significantly improved the sympathetic nervous system compared to listening to loud noises. Lee et al. (2016) reported that inhaling woody essential oil during working or studying increased the level of arousal of the body and improved the psychological stability. These results indicate that forest stimulation through various senses in indoor environments have a positive impact on the body.

Meanwhile, virtual reality (VR) has been recognized as one of the key technologies that lead the fourth industrial revolution (Korea Information Society Development Institute, 2016), and is an artificially created reality through various computer interfaces to make users feel it is like reality or have unusual experiences through multiple senses (sight, sound, touch, force sensing, vestibular sense, etc.) (Brooks et al., 1999). VR has been researched and utilized in various fields, and, for example, VR is utilized in training doctors about surgical techniques to improve the skills of doctors in the field of medicine (Aggarwal et al., 2009). It is also used in social cognition training programs for patients with autism (Kandalaft et al., 2013), and in exposure treatments for those with acrophobia, claustrophobia and arachnophobia (Garcia-Palacios et al., 2002; Powers and Emmelkamp, 2008; Rothbaum et al., 2000). VR is also utilized in military training (Livingston et al., 2002).

As VR has been utilized in various fields for the purpose of education and treatment, it is also expected to solve the problem related to the poor access of people who have walking difficulties to forest and to relieve youths' fear of the forest through indirect forest experiences. However, there has been no study on the effects of forest stimulation using VR on the body. Therefore, it is necessary to identify the effects of forest stimulation using VR on the body first, and 
to conduct studies on those with difficulties in walking and adolescents with fear of forests later. Against this backdrop, this study aimed to identify the effects of visual stimulation through VR forest videos on their stress level.

\section{Materials and Methods}

\section{Subjects and date}

This experiment was conducted on adults aged between 20 and 34, and a total of 40 adults (23 males and 17 females) participated in this experiment. The average age of subjects was $24.4 \pm 2.8$. To ensure they are under the same conditions before measuring heart rate variability (HRV) and hear rate (HR), the following conditions were surveyed before conducting this experiment: mental diseases, physical diseases, experience of certain drugs, etc. They were instructed not to consume caffeine and do excessive exercises prior to this experiment, and this experiment was conducted between 9:00 and 18:00 from November 7 to December 6, 2018.

\section{Methods}

This experiment was conducted in the following order: orientation, wearing equipment, measuring the base line, questionnaire, mental arithmetic stress, watching a VR forest video, questionnaire, and removing equipment (Figure 1). The total time required was 38 minutes. This experiment was carried out in an artificial climate chamber in which temperature, humidity and illuminance can be controlled, and temperature, humidity and illuminance were set at $25^{\circ} \mathrm{C}, 50 \%$ and 500 Lux respectively. Physiological changes were continuously measured from the stage of wearing equipment to the last stage. To measure psychological changes, a questionnaire survey was conducted right after the stage of measuring the base line and that of watching a VR forest video.

\section{Measuring the base line}

To measure the base line of subjects, subjects wore a VR headset on which no video was played and sit in a chair for 5 minutes. The heart rate variability (HRV) and heart rate (HR) of subjects were consecutively measured for 5 minutes, and a questionnaire survey was conducted after the stage of measuring the base line.

\section{Watching a VR forest video}

The stage of watching a VR forest video was conducted to measure physiological and psychological changes caused

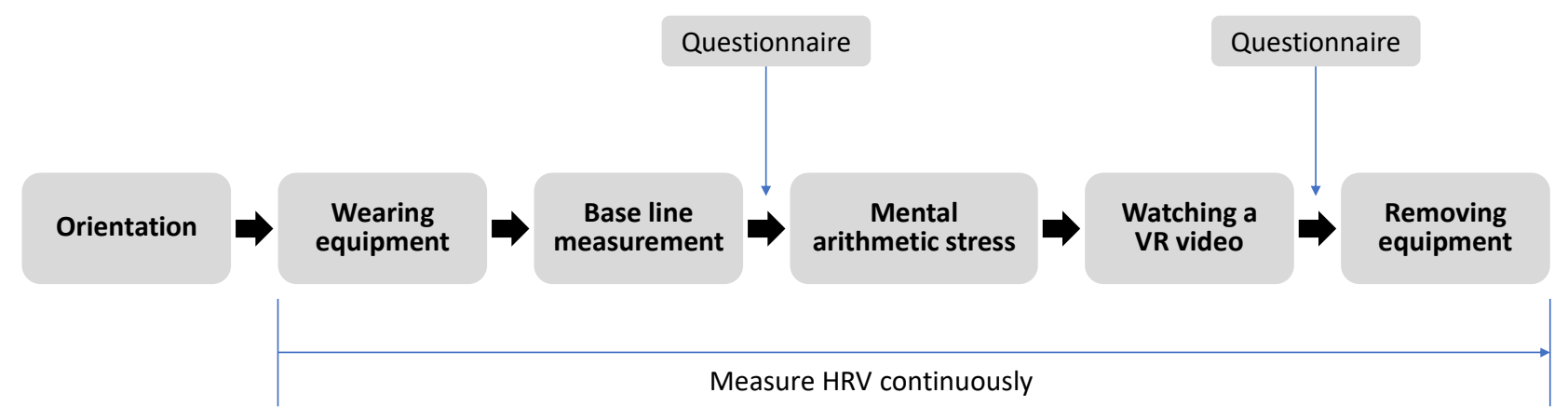

Figure 1. Order of the experiment. 
by watching a VR forest video. The video used in this study was recorded at Minjuji Mountain using a 360-degree camera, and the VR video was created after removing sound (Table 1). Subjects sit in a chair, wearing a VR headset (HTC VIVE PRO, HTC, Taiwan) (Figure 2) and watched the VR forest video for 5 minutes (Figure 2). Their HRV and HR were measured consecutively, and a questionnaire survey was conducted after watching the video.

\section{Mental arithmetic stress}

The stage of mental arithmetic was conducted to cause stress. Subjects wore a VR headset, and questions of multiplying a two-digit number by an one-digit number were displayed on the flat screen of the VR headset. Subjects were instructed to do a mental calculation and say answers. The stage was carried out for 2 minutes. To present questions of the equal difficulty level, questions were created using the table of random sampling numbers.

Table 1. Characteristics of a VR forest video

VR forest video

\begin{tabular}{|c|c|}
\hline Screen & \\
\hline Characteristics & $\begin{array}{l}\text { 5.2k resolution } \\
\text { 360-degree view screen } \\
\text { Sound eliminated in a VR forest video } \\
\text { Seasonal background in a VR forest video is summer. } \\
\text { Forest physiognomy in a VR forest video is mixed forest. } \\
\text { A VR forest video taken } 56 \mathrm{~cm} \text { above the ground. }\end{array}$ \\
\hline
\end{tabular}

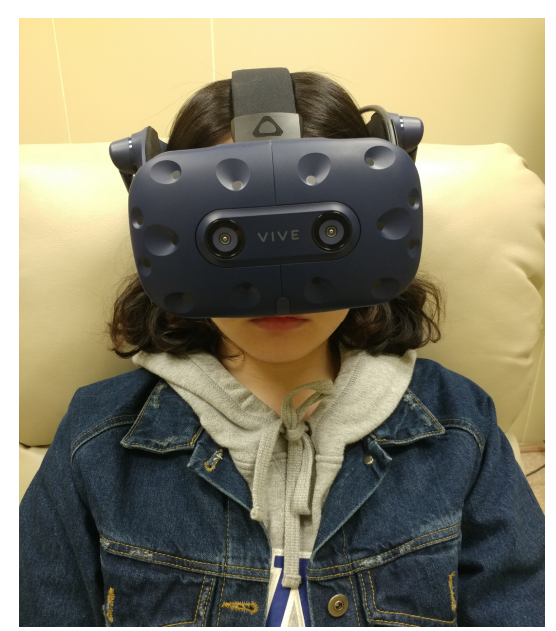

Figure 2. Subject wearing a VR headset during the experiment. 


\section{Measuring tools}

\section{Heart rate variability (HRV)}

Heart rate variability (HRV) is a method of measuring responses of the autonomic nervous system based on variation in the time interval between heartbeats (Kobayashi et al., 1999). In this study, HRV was measured using a portable wearable sensor, and subjects wore the device from the stage of wearing equipment to the last stage to measure HRV consecutively (Figure 3). To measure changes in the activity of the autonomic nervous system, the stress index (SI) and standard deviation of normal to normal RR intervals (SDNN) of subjects were used as an indicator, and to measure changes in the activity of the parasympathetic nervous system, high frequency (HF) was used as an indicator.

\section{(1) SI (stress index)}

The stress index (SI) is a stress analyzing method developed by Baevsky et al. (1984) and is widely used to measure stress based on HRV. The higher the SI, the higher the level of stress.

\section{(2) SDNN (standard deviation of normal to normal RR intervals)}

The standard deviation of normal to normal RR intervals (SDNN) is used as an indicator of the entire heart rate variability during the recorded period, therefore SDNN shows the reactivity of heart rhythm and reflects the activity of the sympathetic and parasympathetic nervous systems at the same time. The higher SDNN, the higher the activity of heart rhythm (Task Force of the European Society of Cardiology and the North American Society of Pacing and Electrophysiology, 1996).

\section{(3) HF (high frequency)}

High frequency $(\mathrm{HF})$ is the range of electromagnetic waves between 0.15 and $0.4 \mathrm{~Hz}$. The higher $\mathrm{HF}$, the more the parasympathetic nervous system is activated, which indicates a stable state (Berntson et al., 1997).

\section{Heart rate (HR)}

In this study, heart rate (HR) was used as an indicator of the sympathetic nervous system. The HR of subjects was measured using a wearable heart rate sensor from the stage of wearing equipment to the last stage continuously (Figure 3 ). The higher the HR, the higher the tension of the body.

\section{(A)}

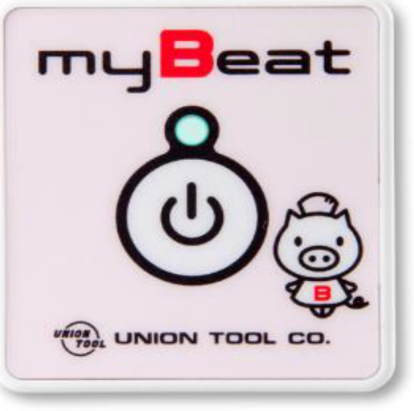

(B)
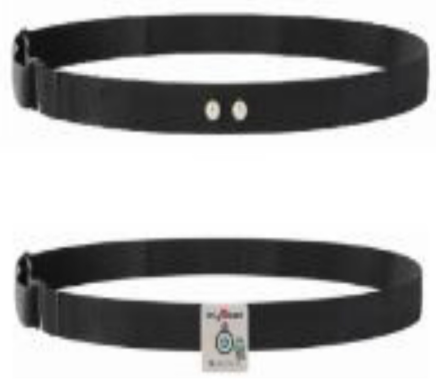

(C)

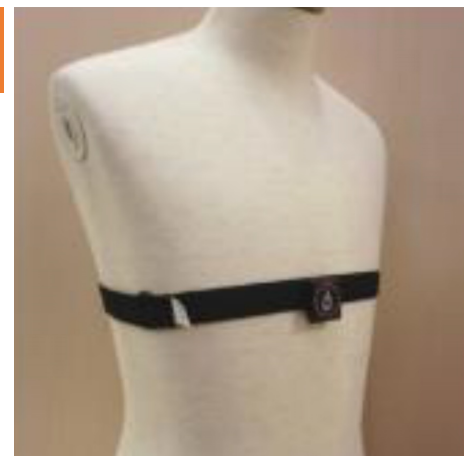

Figure 3. Wearable heart rate sensor from Union Tool Co. (A), electrode sensor connected to a chest strap (B), and position of the strap on the body (C). 


\section{Semantic differential (SD) method}

The semantic differential (SD) method (Osgood et al., 1957) is a method used to assess the image space of humans utilizing adjectives that express their emotions. This study used the following adjectives: 'comfortable-uncomfortable,' 'natural-artificial' and 'soothed-stimulating.' A 13-point scale was used to assess and the higher the score, the higher the comfortable, natural and soothed feelings subjects have. The internal consistency (Cronbach's $\alpha$ ) of this study was .885 .

\section{Profile of mood states (POMS)}

The profile of mood states (POMS) (McNair et al., 1971) is a questionnaire used to assess temporary feelings and emotions, and in this study a simplified version composed of 30 questions and a 5-point scale were used. The POMS is composed of 6 sub scales including tension-anxiety (T-A), depression-dejection (D), anger-hostility (A-H), vigoractivity (V), fatigue-inertia (F) and confusion-bewilderment (C), and each sub scale can be scored. The total mood disturbance (TMD) is calculated by subtracting the score of a positive feeling, vigor-activity, from the sum of the scores of five sub scales of negative feelings including tension-anxiety, depression-dejection, anger-hostility, fatigue-inerti and confusion-bewilderment. The higher the TMD, the higher negative feelings. The internal consistency (Cronbach's $\alpha$ ) of this study was .881 .

\section{Positive affect and negative affect schedule (PANAS)}

The positive affect and negative affect schedule (PANAS) (Watson et al., 1988) is a questionnaire used to assess negative and positive emotions. In this study, the PANAS that was developed by Watson et al. (1988) and validated by Park and Lee (2016) was used. A 5-point Likert scale was used, and the PANAS was composed of a total of 20 questions (10 questions about positive emotions, 10 questions about negative emotions). The internal consistency (Cronbach's $\alpha$ ) of this study was .889 .

\section{Data analysis}

To identify the effects of a VR forest video on the level of stress, subjects' data were compared and analyzed, and the paired sample t-test was conducted on physiological changes. Psychological changes were tested using the Wilcoxon signed rank test, a non-parametric statistical method. Statistical analysis was conducted using IBM SPSS 24.0 (Statistical Package for Social Science, Version 24, SPSS Inc, Illinois, USA), and the significance level was $p<.05$.

\section{Results and Discussion}

\section{Physiological assessment}

This study measured subject's heart rate (HR) and heart rate variability (HRV) in the stages of measuring the base line, mental arithmetic, and watching a VR forest video. In terms of SI, the base line of subjects was $17.3 \pm 0.7 \%$, and the score of metal arithmetic significantly increased to $18.6 \pm 0.6 \%(p<.05$, Figure 4$)$. The score of watching a VR forest video significantly decreased to $16.17 \pm 0.4 \%$ ( $p<.01$, Figure 6 ), which indicates that watching a VR forest video can reduce the stress index that was increased while doing mental arithmetic. In terms of the SDNN, the base line was $24.4 \pm 0.7 \mathrm{msec}$, and the score of mental arithmetic increased to $25.0 \pm 0.7 \mathrm{msec}$ but did not show any significance. The score of watching a VR forest video significantly increased to $26.3 \pm 0.6 \mathrm{msec}(p<.01$, Figure 4$)$, which indicates that watching a VR forest video can increase the level of the SDNN. In terms of HF, the base line was $259.7 \pm 19.2 \mathrm{msec}^{2}$, and the score of mental arithmetic significantly decreased to $222.7 \pm 17.4 \mathrm{msec}^{2}(p<.01$, Figure 4$)$. The score of watch- 
ing a VR forest video significantly increased to $252.9 \pm 16.2 \mathrm{msec}^{2}(p<.05$, Figure 4$)$, which indicates that watching a VR forest video can increase the activity of the parasympathetic nervous system that was decreased while doing mental arithmetic.

Changes in the HR of subjects in the stages of measuring the base line, mental arithmetic, and watching a VR forest video were measured. The base line of subjects was $71.7 \pm 1.5 \mathrm{bpm}$, and the score of mental arithmetic significantly increased to $72.5 \pm 1.4 \mathrm{bpm}(p<.01$, Figure 5). The score of watching a VR forest video significantly decreased to $67.8 \pm 1.3$ $\operatorname{bpm}(p<.01$, Figure 5), which indicates that watching a VR forest video can decrease the heart rate of subjects that was decreased while doing mental arithmetic.

The results of physiological changes show that watching a VR forest video was effective in reducing the stress index and heart rate of subjects, and increasing the activity of the parasympathetic nervous system and the level of the SDNN.

\section{Psychological assessment}

The comfortable, natural and soothed feelings of subjects after watching a VR forest video were measured using the semantic differential (SD) method. In terms of comfortable feeling, the base line of subjects was $7.1 \pm 0.3$, and the score of watching a VR forest video significantly increased to $9.5 \pm 0.3(p<.01$, Figure 6$)$. In terms of natural feeling, the base

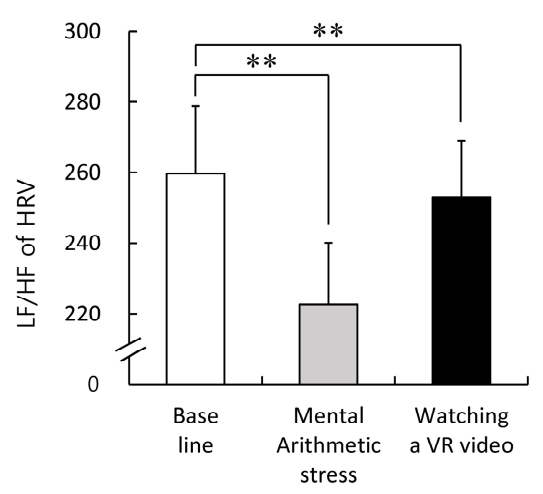

(A) Stress index

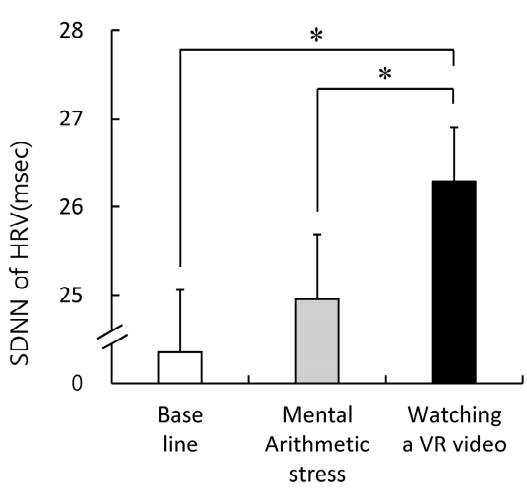

(B) SDNN

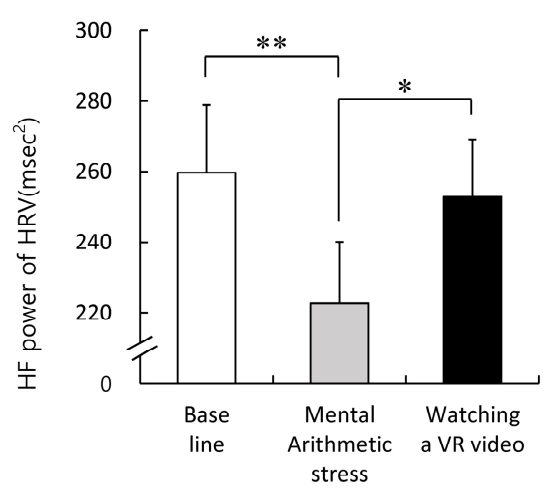

(C) HF

Figure 4. Comparison of heart rate variability in three stages during the experiment. Error bars represent the standard error of the mean $(n=39)$. SDNN = standard deviation of normal to normal RR intervals; HF = high frequency. ${ }^{*} p<.05$, ${ }^{* *} p<.01$ by paired t-test.

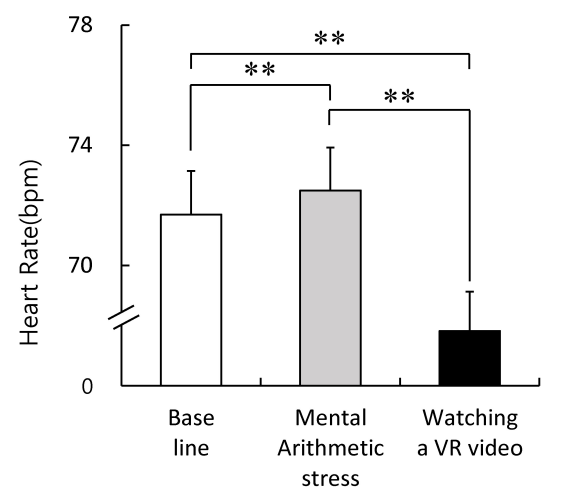

Figure 5. Comparison of heart rate in three stages during the experiment. Error bars represent the standard error of the mean $(n=39) .{ }^{* *} p<.01$ by paired t-test. 
line was $5.7 \pm 0.3$, and the score of watching a VR forest video significantly increased to $9.4 \pm 0.4(p<.01$, Figure 6 ). In terms of soothed feeling, the base line was $6.3 \pm 0.4$, and the score of watching a VR forest video significantly increased to $9.1 \pm 0.4$ ( $p<.01$, Figure 6$)$.

The profile of mood states (POMS) was conducted to measure temporary emotions and feelings including tensionanxiety (T-A), depression-dejection (D), anger-hostility (A-H), vigor (V), fatigue (F), confusion (C) and total mood disturbance (TMD). The base line of tension-anxiety (T-A) was 3.6 \pm 0.6 , and the score of watching a VR forest video significantly decreased to $1.1 \pm 0.3$ ( $p<.01$, Figure 7). The base line of depression-dejection (D) was $2.3 \pm 0.4$, and the score of watching a VR forest video significantly decreased to $0.8 \pm 0.3$ ( $p<.01$, Figure 7$)$. The base line of anger-hostility (A-H) was $1.7 \pm 0.4$, and the score of watching a VR forest video decreased to $0.6 \pm 0.2(p<.01$, Figure 7$)$. The base line of vigor (V) was 2.9 \pm 0.6 , and the score of watching a VR forest video significantly increased to $7.5 \pm 0.9$ ( $p<.01$, Figure 7). The base line of fatigue (F) was 5.7 \pm 0.6 , and the score of watching a VR forest video significantly decreased to $2.2 \pm 0.5$ $(p<.01$, Figure 7 ). The base line of confusion (C) was $3.3 \pm 0.5$, and the score of watching a VR forest video decreased to $2.5 \pm 0.3$, but did not show any significance. The base line of the total mood disturbance (TMD) was $13.6 \pm 2.2$, and the score of watching a VR forest video significantly decreased to $-0.2 \pm 1.6(p<.01$, Figure 7$)$.

The positive affect and negative affect schedule (PANAS) was conducted to measure positive and negative emotions after watching a VR forest video. The base line of positive emotions was $18.5 \pm 1.3$, and the score of watching a VR forest video significantly increased to $23.0 \pm 1.2(p<.01$, Figure 8 ). The base line of negative emotions was $17.0 \pm 1.0$, and the score of watching a VR forest video significantly decreased to $13.0 \pm 0.8(p<.01$, Figure 8$)$.

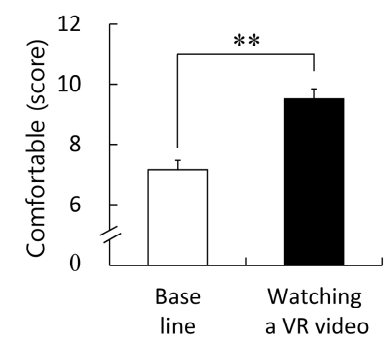

(A) Comfortable

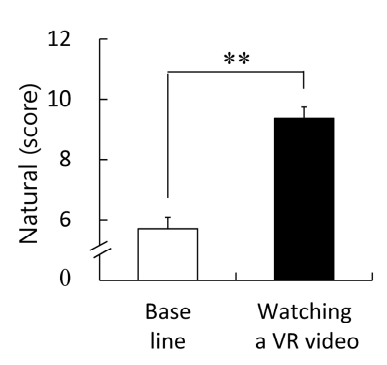

(B) Natural

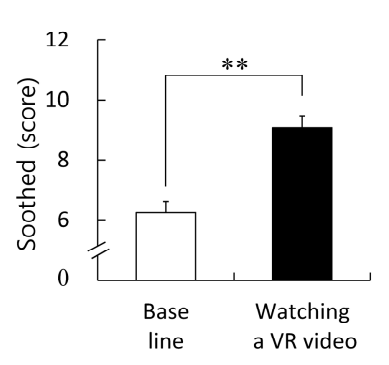

(C) Soothed

Figure 6. Comparison of subjective feelings measured by SD method after watching a VR forest video. Error bars represent the standard error of the mean $(\mathrm{N}=40) .{ }^{* *} p<.01$ by paired Wilcoxon signed-rank test.

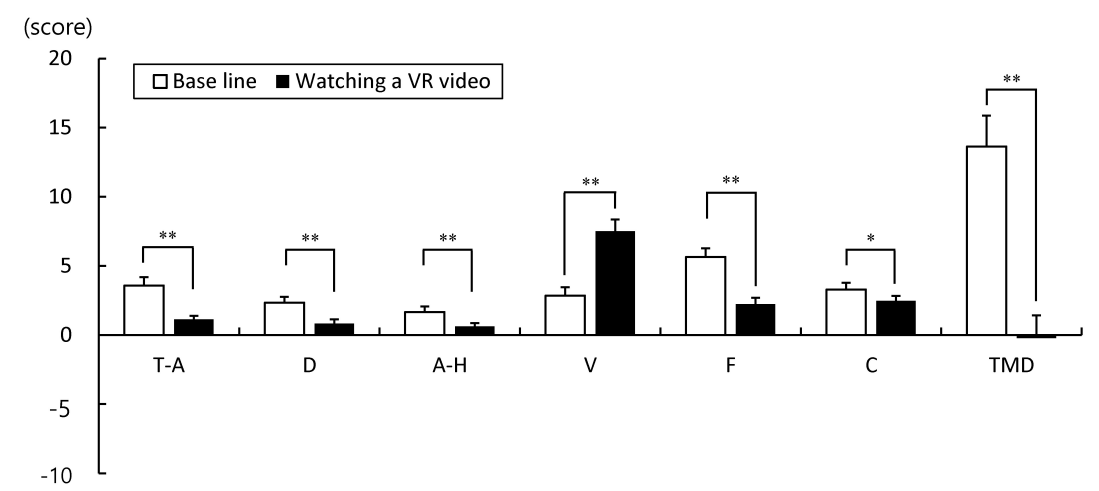

Figure 7. Comparison of profile of mood state after watching a VR forest video. Error bars represent the standard error of the mean $(N=40)$. T-A = tension-anxiety; $D=$ depression-dejection; $A-H=$ anger-hostility; $V=$ vigor; $F=$ fatigue; $\mathrm{C}=$ confusion. ${ }^{*} p<.05,{ }^{* *} p<.01$ by paired Wilcoxon signed-rank test. 


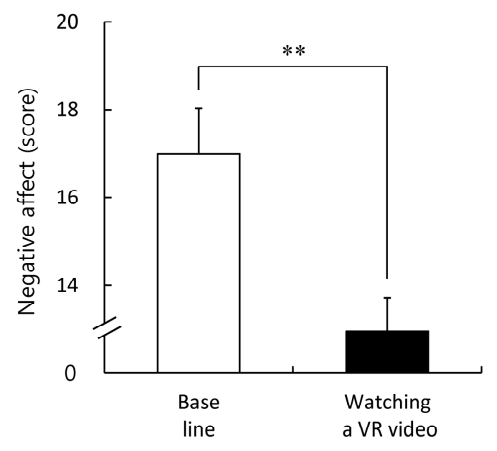

(A) Positive affect

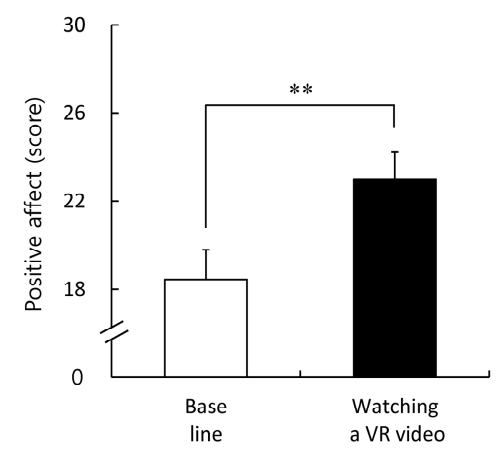

(B) Negative affect

Figure 8. Comparison of positive affect and negative affect schedule after watching a VR forest video. Error bars represent the standard error of the mean $(\mathrm{N}=40) .{ }^{* *} p<.01$ by paired Wilcoxon signed-rank test.

These psychological assessment results show that watching a VR forest video has a positive impact on the psychological state of subjects compared to their base line. The results of the SD test show that watching a VR forest video increased comfortable, natural and soothed feelings, and those of the POMS show that watching a VR forest video reduced the level of tension, depression, fatigue and the TMD and increased the level of vigor among six sub scales compared to the base line of subjects. The results of the PANAS also show that watching a VR forest video increased positive emotions and decreased negative emotions compared to the base line of subjects.

\section{Conclusion}

This study aimed to identify the visual effects of forest healing services provided in indoor environments by measuring the effects of watching a VR forest video on the level of stress through physiological and psychological assessment. Physiological assessment was conducted by measuring heart rate variability (HRV) and heart rate (HR), and it was found that watching a VR forest video reduced the stress index and heart rate of subjects, and increased the activity of the parasympathetic nervous system and the level of the SDNN. Psychological assessment was performed through the profile of mood states (POMS), semantic differential (SD) method and positive affect and negative affect schedule (PANAS), and it was found that subjects had comfortable, natural soothed feelings while watching a VR forest video, and that watching a VR forest video increased the tension, depression, fatigue and total mood disturbance (TMD) of subjects compared to their base line. It was also found that watching a VR forest video reduced negative emotions and increased positive emotions compared to the base line of subjects. These results indicate that watching a VR forest video is effective in reducing the stress index of the body, improving the stability of the body and having a positive impact on the psychological state of humans. These results coincide with those of earlier studies that forest stimulation was effective in reducing stress and improve feelings (Lee, 2017; Jeon and Shin, 2017).

This study was conducted on adults to identify the effects of visual stimulation using a VR forest video, and the results of this study indicated that watching a VR forest video reduced the stress level of adults. These results can be utilized as base data on the visual effects of forest therapy. Since this study was conducted on adults, it will be necessary to conduct additional studies targeting those with walking difficulties and adolescents with fear of forests. It will be also necessary to compare these results with the effects of watching a two-dimensional (2D) forest video to verify the effects of visual stimulation using a forest video. 
There are some limitations in this study. The number of subjects was small and this study targeted a certain age group. Physiological assessment was conducted using the indicators of the autonomic nervous system only. To address these limitations, it will be necessary to target various age groups and use a wide range of physiological assessment indicators. In addition, the questionnaire used in psychological assessment in this study did not measure the curiosity and inquiring mind of subjects towards VR materials. Therefore, various psychological indicators need to be reflected in follow-up studies. Lastly, elements in a VR forest video such as weather, aquatic elements, and degree of closure seem to have a significant impact on the physiological and psychological state of subjects, but these elements were not considered in this study. Therefore, it will be necessary to consider various elements in VR forest videos in follow-up studies.

The results of this study are of great significance. Unlike other earlier studies that utilized 2D images and videos only, this study utilized virtual reality (VR), one of the promising technologies in the fourth industrial revolution. While earlier studies focused on identifying the effects of forest therapy, the purpose of this study was to provide forest welfare for people in the blind spots of forest welfare including those with walking difficulties and adolescents with fear of forests, which is differentiated from earlier studies. The results of this study are expected to contribute to lay a foundation for providing forest welfare for those in the blind spots of forest welfare, and VR, one of the technologies that lead the 4th industrial revolution, can be utilized in various fields.

\section{References}

Aggarwal, R., P. Crochet, A. Dias, A. Misra, P. Ziprin, and A. Darzi. 2009. Development of a virtual reality training curriculum for laparoscopic cholecystectomy. Br. J. Surg. 96(9):1086-1093. https://doi.org/10.1002/bjs.6679

Alvarsson, J.J., S. Wiens, and M.E. Nilsson. 2010. Stress recovery during exposure to nature sound and environmental noise. Int. J. Environ. Res. Public Health 7(3):1036-1046. https://doi.org/10.3390/ijerph7031036

Baevskii, R., O.I. Kirillov, and S.Z. Kletskin. 1984. Mathematical analysis of stress-induced changes in heart rate. Moscow: Nauka.

Berntson, G.G., J.T. Bigger Jr., D.L. Eckberg, P. Grossman, P.G. Kaufmann, M. Malik, H.N. Nagaraja, S.W. Porges, J.P. Saul, P.H. Stone, and M.W. van der Molen. 1997. Heart rate variability: Origins, methods, and interpretive caveats. Psychophysiology 34(6):623-648.

Brooks, F.P. 1999. What's real about virtual reality? IEEE Comput. Graph. Appl. 19(6):16-27. https://doi.org/10.1109/38.799723

Garcia-Palacios, A., H. Hoffman, A. Carlin, T.A. Furness III, and C. Botella. 2002. Virtual reality in the treatment of spider phobia: A controlled study. Behav. Res. Ther. 40(9):983-993. https://doi.org/10.1016/S0005-7967(01)00068-7

Gen, O.I. 2015. Introduction to the new forest healing. Firforest (pp. 222-226). Seoul, Korea: Korea Forest Therapy Forum.

Jeon, J.Y. and C.S. Shin. 2017. Effects of indirect forest experience on human psychology. Korean J. Environ. Ecol. 31(4):420-427. https://doi.org/10.13047/KJEE.2017.31.4.420

Jung, D.W., D.G. Yeom, G.W. Kim, and B.J. Park. 2015. Physiological and psychological relaxing effects of viewing the scenery in the Jangseong Healing Forest. J. Korean Soc. People Plants Environ. 18(5):429-435.

Kandalaft, M.R., N. Didehbani, D.C. Krawczyk, T.T. Allen, and S.B. Chapman. 2013. Virtual reality social cognition training for young adults with high-functioning autism. J. Autism Dev. Disord. 43(1):34-44. https://doi.org/10.1007/s10803-012-1544-6

Kobayashi, H., K. Ishibashi, and H. Noguchi. 1999. Heart rate variability: An index for monitoring and analyzing human autonomic activities. Appl. Human Sci. 18(2):53-59.

Korea Forest Service. 2014. Major business plans for 2014 (pp. 30-33). Daejeon, Korea: Author.

Korea Forest Service. 2018. Forest welfare promotion plans for 2018-2022. (p. 12) Daejeon, Korea: Author. 
Korea Forest Service. 2019. Major business promotion plans for 2019. (p. 131). Daejeon, Korea: Author.

Korea Information Society Development Institute. 2016. Virtual reality (VR) ecosystem status and implications (pp. 1-2). Jincheon-gun, Korea: Author.

Lee, J.W., P.S. Yeon, S.H. Park, and J.W. Kang. 2018. Effects of forest therapy programs on the stress and emotional change of emotional labor workers. J. Korean Inst. For. Recreat. 22(3):15-22

Lee, J.Y. 2017. Experimental study on the health benefits of garden landscape. Int. J. Environ. Res. Public Health 14(7): 829. https://doi.org/10.3390/ijerph14070829

Lee, S.G., D.W. Joung, G.W. Kim, C.H. Park, and B.J. Park. 2016. The influence of inhaling woody essential oil during work on attention-with emphasis on physiological and psychological evaluations -. J. Korean Soc. People Plant Environ. 19(5):465-470.

Livingston, M.A., L.J. Rosenblum, S.J. Julier, D. Brown, Y. Baillot, J. E. Swan II, J.L. Gabbard, and D. Hix. 2002, December. An augmented reality system for military operations in urban terrain [Abstr]. Proceedings of Interservice/ Industry Training, Simulation \& Education Conference (I/ITSEC, p. 89). Orlando, Florida.

McNair, D.M., M. Lorr, and L.F. Droppleman. 1971. Manual for the profile of mood states. San Diego, CA: Educational and Industrial Testing Service.

Osgood, C.E., G.J. Suci, and P.H. Tannenbaum. 1957. The measurement of meaning. Oxford, England: University of Illinois Press.

Park, B.J. 2010. Experimental approach of therapeutic effect of forest recreation activities - Focused on viewing and walking in forest environments -. Doctoral dissertation, Chungnam National University, Daejeon, Korea.

Park, H.S and J.M. Lee. 2016. A validation study of Korean version of PANAS-Revised. Korean J. Psychol. Gen. 35(4): 617-641.

Powers, M.B. and P.M. Emmelkamp. 2008. Virtual reality exposure therapy for anxiety disorders: A meta-analysis. J. Anxiety Disord. 22(3):561-569.

Rothbaum, B.O., L. Hodges, S. Smith, J.H. Lee, and L. Price. 2000. A controlled study of virtual reality exposure therapy for the fear of flying. J. Consult. Clin. Psychol. 68(6):1020-1026.

Task Force of the European Society of Cardiology and the North American Society of Pacing and Electrophysiology. 1996. Heart rate variability: Standards of measurement, physiological interpretation and clinical use. Circulation 93(5): 1043-1065. https://doi.org/10.1161/01.CIR.93.5.1043

Watson, D., L.A. Clark, and A. Tellegen. 1988. Development and validation of brief measures of positive and negative affect: The PANAS scales. J. Pers. Soc. Psychol. 54(6):1063-1070.

Yeom, D.G. 2015. A study on the analysis of the characteristics of Korea forest welfare facilities and the content development of the associated-program for life cycle stage. Master's thesis, Chungnam National University, Daejeon, Korea. 